\title{
The disc separation and the eigenvalue distribution of the Schur complement of nonstrictly diagonally dominant matrices
}

\author{
Cheng-yi Zhang ${ }^{1 *}$, Weiwei Wang ${ }^{1}$, Shuanghua Luo ${ }^{1}$ and Jianxing Zhao ${ }^{2 *}$
}

\author{
"Correspondence: \\ cyzhang08@126.com; \\ zjx810204@163.com \\ 'School of Science, Xi'an \\ Polytechnic University, Xi'an, \\ Shaanxi 710048, China \\ ${ }^{2}$ College of Science, Guizhou Minzu \\ University, Guiyang, Guizhou \\ 550025, China
}

\begin{abstract}
The result on the Geršgorin disc separation from the origin for strictly diagonally dominant matrices and their Schur complements in (Liu and Zhang in SIAM J. Matrix Anal. Appl. 27(3):665-674, 2005) is extended to nonstrictly diagonally dominant matrices and their Schur complements, showing that under some conditions the separation of the Schur complement of a nonstrictly diagonally dominant matrix is greater than that of the original grand matrix. As an application, the eigenvalue distribution of the Schur complement is discussed for nonstrictly diagonally dominant matrices to derive some significant conclusions. Finally, some examples are provided to show the effectiveness of theoretical results.
\end{abstract}

MSC: 15A06; 15A15; 15A48

Keywords: Geršgorin disc separation; Schur complement; nonstrictly diagonally dominant matrices; eigenvalue distribution

\section{Introduction}

Let $A=\left(a_{i j}\right) \in \mathbb{C}^{n \times n}$ with $R_{i}(A)=\sum_{j=1, j \neq i}^{n}\left|a_{i j}\right|$ for all $i \in\langle n\rangle$ define the disc separation of $A$ by the quantities $\left|a_{i i}\right|-R_{i}(A)$ that measure the separations of the discs

$$
\left|z-a_{i i}\right| \leq R_{i}(A), \quad i=1,2, \ldots, n,
$$

from the origin and give estimate $\min _{1 \leq i \leq n}\left|a_{i i}\right|-R_{i}(A)$ of the absolute value of the shortest eigenvalue (the eigenvalue with the smallest absolute value, see [1]) of the strictly diagonally dominant matrix $A \in \mathbb{C}^{n \times n}$.

In 2005, Liu and Zhang [1] firstly studied the disc separation of the Schur complements of strictly diagonally dominant matrices. More precisely, they compared a disc separation of the Schur complement to that of the original matrix and showed that each Geršgorin disc of the Schur complement is paired with a particular Geršgorin disc of the original matrix; the latter is further from the origin than the former. Their result is as follows.

Theorem 1 (see [1]) Given an $n \times n$ strictly diagonally dominant matrix $A=\left(a_{i j}\right)$ and two sets $\alpha=\left\{i_{1}, i_{2}, \ldots, i_{m}\right\} \subset\langle n\rangle=\{1,2, \ldots, n\}$ and $\alpha^{\prime}=\langle n\rangle-\alpha=\left\{j_{1}, j_{2}, \ldots, j_{l}\right\} \subset\langle n\rangle$ with

(c) The Author(s) 2017. This article is distributed under the terms of the Creative Commons Attribution 4.0 International License (http://creativecommons.org/licenses/by/4.0/), which permits unrestricted use, distribution, and reproduction in any medium, provided you give appropriate credit to the original author(s) and the source, provide a link to the Creative Commons license, and indicate if changes were made. 
$m+l=n, l e t$

$$
\omega_{j_{t}}=\min _{1 \leq \nu \leq m} \frac{\left|a_{i_{v} i_{v}}\right|-R_{i_{v}}}{\left|a_{i_{v} i_{v}}\right|} \sum_{u=1}^{m}\left|a_{j_{t} i_{u}}\right|
$$

for all $j_{t} \in \alpha^{\prime}$, and define $A / \alpha=\left(\widetilde{a}_{j_{t}, j_{s}}\right)$, then

$$
\left|\widetilde{a}_{j_{t}, j_{t}}\right|-R_{j_{t}}(A / \alpha) \geq\left|a_{j_{t}, j_{t}}\right|-R_{j_{t}}(A)+\omega_{j_{t}} \geq\left|a_{j_{t}, j_{t}}\right|-R_{j_{t}}(A)>0
$$

and

$$
\left|\tilde{a}_{j_{t}, j_{t}}\right|+R_{j_{t}}(A / \alpha) \leq\left|a_{j_{t}, j_{t}}\right|+R_{j_{t}}(A)-\omega_{j_{t}} \leq\left|a_{j_{t}, j_{t}}\right|+R_{j_{t}}(A) .
$$

On the other hand, Liu and Zhang [1] also improved the result of Theorem 1 in [2] when $A \in S D_{n} \subset H_{n}^{S}$ and established the new result on the eigenvalue distribution for the Schur complements of strictly diagonally dominant matrices with real diagonal entries.

Theorem 2 (see [1]) Let $A=\left(a_{i j}\right)$ be an $n \times n$ strictly diagonally dominant matrix with real diagonal entries, and $\alpha \subset\langle n\rangle$. Then $A / \alpha$ and $A\left(\alpha^{\prime}\right)$ have the same number of eigenvalues whose real parts are greater (less) than $w$ (resp. $-w)$, where $\alpha^{\prime}=\langle n\rangle-\alpha$ and

$$
w=\min _{j \in \alpha^{\prime}}\left[\left|a_{j j}\right|-R_{j}(A)+\min _{i \in \alpha} \frac{\left|a_{i i}\right|-R_{i}(A)}{\left|a_{i i}\right|} \sum_{i \in \alpha}\left|a_{j i}\right|\right] .
$$

In this paper, we will generalize the result on the Geršgorin disc separation from the origin for strictly diagonally dominant matrices and their Schur complements in [1] to nonstrictly diagonally dominant matrices and their Schur complements, showing that under some conditions the separation of the Schur complement of a nonstrictly diagonally dominant matrix is greater than that of the original grand matrix. As an application, we continue discussing the eigenvalue distribution of the Schur complements for nonstrictly diagonally dominant matrices to derive some significant conclusions.

The paper is organized as follows. Some notations and preliminary results about nonstrictly diagonally dominant matrices are given in Section 2. Some results on the Geršgorin disc separation from the origin are established in Section 3 for nonstrictly diagonally dominant matrices and their Schur complements. The eigenvalue distribution of the Schur complements is discussed in Section 4 for nonstrictly diagonally dominant matrices to derive some significant conclusions. Some examples are provided in Section 5 to show the effectiveness of theoretical results. Conclusions are given in Section 6.

\section{Preliminaries}

In this section we give some notions and preliminary results about special matrices that are used in this paper.

$\mathbb{C}^{m \times n}\left(\mathbb{R}^{m \times n}\right)$ will be used to denote the set of all $m \times n$ complex (real) matrices.

$\mathbb{Z}$ denotes the set of all integers. Let $\alpha \subseteq\langle n\rangle=\{1,2, \ldots, n\} \subset \mathbb{Z} .|\alpha|$ denotes the cardinality of the set $\alpha$. For nonempty index sets $\alpha, \beta \subseteq\langle n\rangle, A(\alpha, \beta)$ is the submatrix of $A \in \mathbb{C}^{n \times n}$ with row indices in $\alpha$ and column indices in $\beta$. The submatrix $A(\alpha, \alpha)$ is abbreviated to $A(\alpha)$. 
Let $A \in \mathbb{C}^{n \times n}, \alpha \subset\langle n\rangle$ and $\alpha^{\prime}=\langle n\rangle-\alpha$. If $A(\alpha)$ is nonsingular, the matrix

$$
A / \alpha=A\left(\alpha^{\prime}\right)-A\left(\alpha^{\prime}, \alpha\right)[A(\alpha)]^{-1} A\left(\alpha, \alpha^{\prime}\right)
$$

is called the Schur complement with respect to $A(\alpha)$, indices in both $\alpha$ and $\alpha^{\prime}$ are arranged with increasing order. We shall confine ourselves to the nonsingular $A(\alpha)$ as far as $A / \alpha$ is concerned.

A matrix $A=\left(a_{i j}\right) \in \mathbb{R}^{n \times n}$ is called nonnegative if $a_{i j} \geq 0$ for all $i, j \in\langle n\rangle$. A matrix $A=$ $\left(a_{i j}\right) \in \mathbb{R}^{n \times n}$ is called a $Z$-matrix if $a_{i j} \leq 0$ for all $i \neq j$. We will use $Z_{n}$ to denote the set of all $n \times n Z$-matrices. A matrix $A=\left(a_{i j}\right) \in Z_{n}$ is called an $M$-matrix if $A$ can be expressed in the form $A=s I-B$, where $B \geq 0$, and $s \geq \rho(B)$, the spectral radius of $B$. If $s>\rho(B), A$ is called a nonsingular $M$-matrix $M_{n}$ and $M_{n}^{\bullet}$ will be used to denote the set of all $n \times n$ $M$-matrices and the set of all $n \times n$ nonsingular $M$-matrices, respectively (see [3]).

The comparison matrix of a given matrix $A=\left(a_{i j}\right) \in \mathbb{C}^{n \times n}$, denoted by $\mu(A)=\left(\mu_{i j}\right)$, is defined by

$$
\mu_{i j}= \begin{cases}\left|a_{i i}\right|, & \text { if } i=j, \\ -\left|a_{i j}\right|, & \text { if } i \neq j .\end{cases}
$$

It is clear that $\mu(A) \in Z_{n}$ for a matrix $A \in \mathbb{C}^{n \times n}$. A matrix $A=\left(a_{i j}\right) \in \mathbb{C}^{n \times n}$ is called a general $H$-matrix if $\mu(A) \in M_{n}$. If $\mu(A) \in M_{n}^{\bullet}$, $A$ is called an invertible $H$-matrix. $H_{n}$ and $H_{n}^{I}$ will denote the set of all $n \times n$ general $H$-matrices and the set of all $n \times n$ invertible $H$-matrices, respectively (see [4]).

Lemma 1 (see [2]) If $A \in H_{n}^{I}$, then

$$
[\mu(A)]^{-1} \geq\left|A^{-1}\right| \geq 0 .
$$

For $n \geq 2$, an $n \times n$ complex matrix $A$ is reducible if there exists an $n \times n$ permutation matrix $P$ such that

$$
P A P^{T}=\left[\begin{array}{cc}
A_{11} & A_{12} \\
0 & A_{22}
\end{array}\right],
$$

where $A_{11}$ is an $r \times r$ submatrix and $A_{22}$ is an $(n-r) \times(n-r)$ submatrix, where $1 \leq r<n$. If no such permutation matrix exists, then $A$ is called irreducible. If $A$ is a $1 \times 1$ complex matrix, then $A$ is irreducible if its single entry is nonzero, and reducible otherwise.

A matrix $A \in \mathbb{C}^{n \times n}$ is called diagonally dominant by row if

$$
\left|a_{i i}\right| \geq \sum_{j=1, j \neq i}^{n}\left|a_{i j}\right|
$$

holds for all $i \in\langle n\rangle$. If inequality in (9) holds strictly for all $i \in\langle n\rangle, A$ is called strictly diagonally dominant by row. If $A$ is irreducible and the inequality in (9) holds strictly for at least one $i \in\langle n\rangle, A$ is called irreducibly diagonally dominant by row. If (9) holds with equality for all $i \in\langle n\rangle, A$ is called diagonally equipotent by row. If (9) holds with equality for at least one $i \in\langle n\rangle, A$ is called nonstrictly diagonally dominant. 
$D_{n}\left(S D_{n}, I D_{n}\right)$ and $D E_{n}$ will be used to denote the sets of all $n \times n$ (strictly, irreducibly) diagonally dominant matrices and the set of all $n \times n$ diagonally equipotent matrices, respectively.

Lemma 2 (see [5]) Let $A \in D_{n}$. Then $A$ is singular if and only if there exists a singular principal submatrix in $A$.

Lemma 3 (see [5]) Let $A \in D_{n}$. Then $A \in H_{n}^{I}$ if and only if $A$ has either one zero principal submatrix or one irreducibly diagonally equipotent principal submatrix. Furthermore, if $A \in D_{n} \cap Z_{n}$, then $A \in M_{n}^{\bullet}$ if and only if $A$ has either one zero principal submatrix or one irreducibly diagonally equipotent principal submatrix.

Lemma 4 (see Lemma 3.4 in [6]) Given a matrix $A \in D_{n}$ and a set $\alpha=\langle n\rangle-\alpha^{\prime} \subseteq\langle n\rangle$, if $A(\gamma)$ is the largest diagonally equipotent principal submatrix of $A(\alpha)$ for $\gamma=\alpha-\gamma^{\prime} \subseteq \alpha$, then $A / \alpha=A\left(\alpha^{\prime} \cup \gamma^{\prime}\right) / \gamma^{\prime}$, where

$$
A\left(\alpha^{\prime} \cup \gamma^{\prime}\right)=\left[\begin{array}{cc}
A\left(\gamma^{\prime}\right) & A\left(\gamma^{\prime}, \alpha^{\prime}\right) \\
A\left(\alpha^{\prime}, \gamma^{\prime}\right) & A\left(\alpha^{\prime}\right)
\end{array}\right] .
$$

Lemma 5 (see [1]) Let $A \in C^{n \times n}$ be partitioned as

$$
A=\left(\begin{array}{ll}
a_{11} & A_{12} \\
A_{21} & A_{22}
\end{array}\right) \text {, }
$$

where $A_{21}=\left(a_{21}, a_{31}, \ldots, a_{n 1}\right)^{T}$ and $A_{12}=\left(a_{12}, a_{13}, \ldots, a_{1 n}\right)$. If $A_{22}$ is nonsingular, then

$$
\frac{\operatorname{det} A}{\operatorname{det} A_{22}}=a_{11}-\left(a_{12}, a_{13}, \ldots, a_{1 n}\right)\left[A_{22}\right]^{-1}\left(\begin{array}{c}
a_{21} \\
\vdots \\
a_{n 1}
\end{array}\right) \text {. }
$$

\section{The disc separation of the Schur complement of nonstrictly diagonally dominant matrices}

In this section, we will establish some results on the Geršgorin disc separation from the origin for nonstrictly diagonally dominant matrices and their Schur complements such that under some conditions the separation of the Schur complement of a nonstrictly diagonally dominant matrix is greater than that of the original grand matrix. Firstly, the following lemma will be used in the rest of this subsection.

Lemma 6 Let $A=\left(a_{i j}\right) \in D_{n}$ be nonsingular, and two sets $\alpha=\left\{i_{1}, i_{2}, \ldots, i_{m}\right\} \subset\langle n\rangle$ and $\alpha^{\prime}=$ $\langle n\rangle-\alpha=\left\{j_{1}, j_{2}, \ldots, j_{l}\right\} \subset\langle n\rangle$ with $m+l=n$. For any $j_{t} \in \alpha^{\prime}$, denote

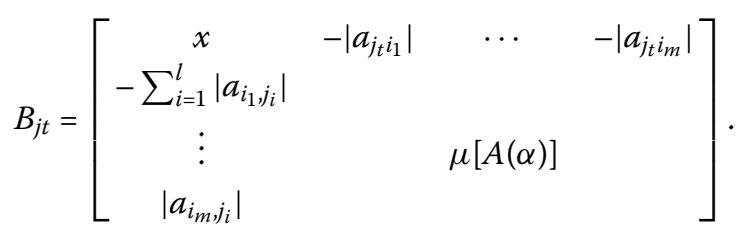

Then $B_{j t}$ is doubly diagonally dominant [7] if and only if

$$
x \geq \max _{1 \leq \omega \leq m} \frac{R_{i_{\omega}}(A)}{\left|a_{i_{\omega} i_{\omega}}\right|} .
$$


When inequality (12) holds, $B_{j t}$ is an M-matrix of order $m+1$, and thus, $\operatorname{det} B_{j t} \geq 0$. Furthermore, if the strict inequality (12) holds and $\left|a_{i_{s} i_{s}}\right|>R_{i_{s}}(A)$ for all $i_{s} \in \alpha$, then $B_{j t}$ is a nonsingular M-matrix of order $m+1$, and thus, $\operatorname{det} B_{j t}>0$.

Proof Similar to the proof of Lemma 4 in [1], we can easily derive the conclusion of this lemma.

Theorem 3 Let $A=\left(a_{i j}\right) \in D_{n}$ be nonsingular, and two sets $\alpha=\left\{i_{1}, i_{2}, \ldots, i_{m}\right\} \subset\langle n\rangle$ and $\alpha^{\prime}=\langle n\rangle-\alpha=\left\{j_{1}, j_{2}, \ldots, j_{l}\right\} \subset\langle n\rangle$ with $m+l=n$, define $A / \alpha=\left(\widetilde{a}_{j_{t}, j_{s}}\right)$ and $\omega_{j_{t}}$ as in $(1)$, if

$$
\left|a_{j_{t}, j_{t}}\right|>R_{j_{t}}(A)
$$

for all $j_{t} \in \alpha^{\prime}$, then both (2) and (3) hold.

Proof Since $A \in D_{n}$ and is nonsingular, Lemma 2 indicates that $A(\alpha)$ is nonsingular. As a result, $A / \alpha=\left(\widetilde{a}_{j_{t}, j_{s}}\right)$ exists. According to definition (5) of the Schur complement matrix $A / \alpha$, we have the off-diagonal entries

$$
\begin{aligned}
& \tilde{a}_{j_{l}, j_{t}}=a_{j_{l}, j_{t}}-\left[\left(a_{j_{l}, i_{1}}, a_{j_{l}, i_{2}}, \ldots, a_{j_{l}, i_{k}}\right)[A(\alpha)]^{-1}\left(\begin{array}{c}
a_{i_{1}, j_{t}} \\
a_{i_{2}, j_{t}} \\
\vdots \\
a_{i_{k}, j_{t}}
\end{array}\right)\right], \\
& l, t=1,2, \ldots, m,
\end{aligned}
$$

and the diagonal entries

$$
\begin{aligned}
& \tilde{a}_{j_{l}, j_{l}}=a_{j_{l}, j_{l}}-\left[\left(a_{j_{l}, i_{1}}, a_{j_{l}, i_{2}}, \ldots, a_{j_{l}, i_{k}}\right)[A(\alpha)]^{-1}\left(\begin{array}{c}
a_{i_{1}, j_{l}} \\
a_{i_{2}, j_{l}} \\
\vdots \\
a_{i_{k}, j_{l}}
\end{array}\right)\right], \\
& l=1,2, \ldots, m,
\end{aligned}
$$

of $A / \alpha$. The conclusion of this theorem will be proved by proving the following two cases:

(i) If $A(\alpha) \in H_{|\alpha|}^{I}$, Lemma 1 gives

$$
\{\mu[A(\alpha)]\}^{-1} \geq\left|[A(\alpha)]^{-1}\right| \geq 0 .
$$

Then from (14), (15), (16) and Lemma 5, we have

$$
\begin{aligned}
\left|\tilde{a}_{j_{t}, j_{t}}\right|-R_{j_{t}}(A / \alpha) & =\left|\tilde{a}_{j_{t}, j_{t}}\right|-\sum_{i=1, i \neq t}^{l}\left|\tilde{a}_{j_{t}, j_{i}}\right| \\
& =\left|\left[a_{j_{t}, j_{t}}-\left(a_{j_{t}, i_{1}}, \ldots, a_{j_{t}, i_{k}}\right)[A(\alpha)]^{-1}\left(\begin{array}{c}
a_{i_{1}, j_{t}} \\
a_{i_{2}, j_{t}} \\
\vdots \\
a_{i_{k}, j_{t}}
\end{array}\right)\right]\right|
\end{aligned}
$$




$$
\begin{aligned}
& -\sum_{i=1, i \neq t}^{l}\left|\left[a_{j_{l}, j_{i}}-\left(a_{j_{l}, i_{1}}, \ldots, a_{j_{l}, i_{m}}\right)[A(\alpha)]^{-1}\left(\begin{array}{c}
a_{i_{1}, j_{i}} \\
a_{i_{2}, j_{i}} \\
\vdots \\
a_{i_{k}, j_{i}}
\end{array}\right)\right]\right| \\
& \geq\left|a_{j l, j l}\right|-\sum_{i=1, i \neq t}^{l}\left|a_{j l, j}\right| \\
& -\sum_{i=1}^{l}\left|\left[\left(a_{j_{t}, i_{1}}, a_{j_{t}, i_{2}}, \ldots, a_{j_{t}, i_{m}}\right)[A(\alpha)]^{-1}\left(\begin{array}{c}
a_{i_{1}, j_{i}} \\
a_{i_{2}, j_{i}} \\
\vdots \\
a_{i_{m}, j_{i}}
\end{array}\right)\right]\right| \\
& \geq\left|a_{j t, j t}\right|-\sum_{i=1, i \neq t}^{l}\left|a_{j t, j i}\right| \\
& -\sum_{i=1}^{l}\left[\left(\left|a_{j_{t}, i_{1}}\right|,\left|a_{j_{t}, i_{2}}\right|, \ldots,\left|a_{j_{t}, i_{m}}\right|\right)\left|[A(\alpha)]^{-1}\right|\left(\begin{array}{c}
\left|a_{i_{1}, j_{i}}\right| \\
\left|a_{i_{2}, j_{i}}\right| \\
\vdots \\
\left|a_{i_{m}, j_{i}}\right|
\end{array}\right)\right] \\
& \geq\left|a_{j t, j t}\right|-\sum_{i=1, i \neq t}^{l}\left|a_{j t, j_{i}}\right| \\
& -\sum_{i=1}^{l}\left[\left(\left|a_{j_{t}, i_{1}}\right|,\left|a_{j_{t}, i_{2}}\right|, \ldots,\left|a_{j_{t}, i_{m}}\right|\right)\{\mu[A(\alpha)]\}^{-1}\left(\begin{array}{c}
\left|a_{i_{1}, j_{i}}\right| \\
\left|a_{i_{2}, j_{i}}\right| \\
\vdots \\
\left|a_{i_{m}, j_{i}}\right|
\end{array}\right)\right] \\
& =\left|a_{j_{t}, j_{t}}\right|-R_{j_{t}}(A)+\sum_{u=1}^{l}\left|a_{j_{t} i_{u}}\right|+\omega_{j_{t}}-\omega_{j_{t}} \\
& -\sum_{i=1}^{l}\left[\left(\left|a_{j_{t}, i_{1}}\right|,\left|a_{j_{t}, i_{2}}\right|, \ldots,\left|a_{j_{t}, i_{m}}\right|\right)\{\mu[A(\alpha)]\}^{-1}\left(\begin{array}{c}
\left|a_{i_{1}, j_{i}}\right| \\
\left|a_{i_{2}, j_{i}}\right| \\
\vdots \\
\left|a_{i_{m}, j_{i}}\right|
\end{array}\right)\right] \\
& =\left|a_{j t, j t}\right|-R_{j t}(A)+\omega_{j t}+\sum_{u=1}^{m}\left|a_{j t i_{u}}\right|-\omega_{j t} \\
& -\sum_{i=1}^{l}\left[\left(\left|a_{j_{t}, i_{1}}\right|,\left|a_{j_{t}, i_{2}}\right|, \ldots,\left|a_{j_{t}, i_{m}}\right|\right)\{\mu[A(\alpha)]\}^{-1}\left(\begin{array}{c}
\left|a_{i_{1}, j_{i}}\right| \\
\left|a_{i_{2}, j_{i}}\right| \\
\vdots \\
\left|a_{i_{m}, j_{i}}\right|
\end{array}\right)\right] \\
& =\left|a_{j_{t}, j_{t}}\right|-R_{j_{t}}(A)+\omega_{j_{t}}+\frac{\operatorname{det} B_{j_{t}}}{\operatorname{det} \mu[A(\alpha)]},
\end{aligned}
$$

where

$$
B_{j_{t}}=\left(\begin{array}{cc}
\sum_{u=1}^{m}\left|a_{j t i_{u}}\right|-\omega_{j_{t}} & h^{T} \\
g & \mu[A(\alpha)]
\end{array}\right)_{(m+1) \times(m+1)},
$$




$$
\begin{aligned}
& g=\left(-\sum_{i=1}^{l}\left|a_{i_{1}, j_{i}}\right|, \ldots,-\sum_{i=1}^{l}\left|a_{i_{m}, j_{i}}\right|\right)^{T}, \\
& h=\left(-\left|a_{j_{l}, i_{1}}\right|, \ldots,-\left|a_{j_{l}, i_{m}}\right|\right)^{T} .
\end{aligned}
$$

It is clear that $B_{j t} \in Z_{m+1}$. In Lemma 6, we set

$$
x=\sum_{u=1}^{m}\left|a_{j t i_{u}}\right|-\omega_{j t} \geq \max _{1 \leq i_{\omega} \leq m} \frac{R_{i_{\omega}}(A)}{\left|a_{i_{\omega} i_{\omega}}\right|} .
$$

According to Lemma $6, \operatorname{det} B_{j_{t}}=\operatorname{det} \mu\left(B_{j_{t}}\right) \geq 0$. Continuing (17),

$$
\begin{aligned}
\left|\tilde{a}_{j_{t}, j_{t}}\right|-R_{j_{t}}(A / \alpha) & \geq\left|a_{j_{t}, j_{t}}\right|-R_{j_{t}}(A)+\omega_{j_{t}}+\frac{\operatorname{det} B_{j_{t}}}{\operatorname{det} \mu[A(\alpha)]} \\
& \geq\left|a_{j_{t}, j_{t}}\right|-R_{j_{t}}(A)+\omega_{j_{t}} \geq\left|a_{j_{t}, j_{t}}\right|-R_{j_{t}}(A)>0 .
\end{aligned}
$$

Similarly,

$$
\begin{aligned}
\left|\tilde{a}_{j_{t}, j_{t}}\right|+R_{j_{t}}(A / \alpha) & \leq\left|a_{j_{t}, j_{t}}\right|+R_{j_{t}}(A)-\omega_{j_{t}}-\frac{\operatorname{det} B_{j_{t}}}{\operatorname{det} \mu[A(\alpha)]} \\
& \leq\left|a_{j_{t}, j_{t}}\right|+R_{j_{t}}(A)-\omega_{j_{t}} \leq\left|a_{j_{t}, j_{t}}\right|-R_{j_{t}}(A) .
\end{aligned}
$$

This completes the proof of case (i).

Next, we prove case (ii). Assume $A(\alpha) \notin H_{|\alpha|}^{I}$, it then follows from Lemma 3 that $A(\alpha)$ has at least one diagonally equipotent principal submatrix. Let $A(\gamma)$ be the largest diagonally equipotent principal submatrix of the matrix $A(\alpha)$ for $\gamma=\alpha-\gamma^{\prime} \subseteq \alpha$. Then $A\left(\gamma^{\prime}\right)$ has no diagonally equipotent principal submatrix and hence $A\left(\gamma^{\prime}\right) \in H_{\left|\gamma^{\prime}\right|}^{I}$ from Lemma 3. Since $A \in D_{n}$ and $A(\gamma)$ is the largest diagonally equipotent principal submatrix of the matrix $A(\alpha)$, it follows from Lemma 4 that $A / \alpha=A\left(\alpha^{\prime} \cup \gamma^{\prime}\right) / \gamma^{\prime}$, where $\alpha^{\prime}=\langle n\rangle-\alpha \subseteq\langle n\rangle$ and $A\left(\alpha^{\prime} \cup \gamma^{\prime}\right)$ is given in (10). Since $A\left(\gamma^{\prime}\right) \in H_{\left|\gamma^{\prime}\right|}^{I} \cap D_{\left|\gamma^{\prime}\right|}$, it follows from the proof of case (i) that both (2) and (3) hold, which shows that the proof of case (ii) is completed. This completes the proof.

Theorem 4 Given a matrix $A=\left(a_{i j}\right) \in D_{n}$ and two sets $\alpha=\left\{i_{1}, i_{2}, \ldots, i_{m}\right\} \subset\langle n\rangle$ and $\alpha^{\prime}=$ $\langle n\rangle-\alpha=\left\{j_{1}, j_{2}, \ldots, j_{l}\right\} \subset\langle n\rangle$ with $m+l=n$, define $\omega_{j_{t}}$ as in (1) and $A / \alpha=\left(\tilde{a}_{j_{t}, j_{s}}\right)$, if $\omega_{j_{t}}>0$ for all $j_{t} \in \alpha^{\prime}$, then

$$
\left|\widetilde{a}_{j_{t}, j_{t}}\right|-R_{j_{t}}(A / \alpha) \geq\left|a_{j_{t}, j_{t}}\right|-R_{j_{t}}(A)+\omega_{j_{t}}>\left|a_{j_{t}, j_{t}}\right|-R_{j_{t}}(A) \geq 0
$$

and

$$
\left|\widetilde{a}_{j_{t}, j_{t}}\right|+R_{j_{t}}(A / \alpha) \leq\left|a_{j_{t}, j_{t}}\right|+R_{j_{t}}(A)-\omega_{j_{t}}<\left|a_{j_{t}, j_{t}}\right|+R_{j_{t}}(A)
$$

Proof Using the same proof method as the one of Theorem 3, the conclusion of this theorem is obtained immediately. 
Theorem 5 Given a matrix $A=\left(a_{i j}\right) \in D_{n}$ and two sets $\alpha=\left\{i_{1}, i_{2}, \ldots, i_{m}\right\} \subset\langle n\rangle$ and $\alpha^{\prime}=$ $\langle n\rangle-\alpha=\left\{j_{1}, j_{2}, \ldots, j_{l}\right\} \subset\langle n\rangle$ with $m+l=n$, define $\omega_{j_{t}}$ as in $(1)$, if $A(\alpha)$ is nonsingular, then $A / \alpha=\left(\widetilde{a}_{j t, j_{s}}\right)$ satisfies

$$
\left|\widetilde{a}_{j t, j t}\right|-R_{j_{t}}(A / \alpha) \geq\left|a_{j t, j t}\right|-R_{j_{t}}(A)+\omega_{j_{t}} \geq\left|a_{j_{t}, j_{t}}\right|-R_{j t}(A) \geq 0
$$

and

$$
\left|\widetilde{a}_{j t, j t}\right|+R_{j t}(A / \alpha) \leq\left|a_{j_{t}, j_{t}}\right|+R_{j t}(A)-\omega_{j_{t}} \leq\left|a_{j_{t}, j_{t}}\right|+R_{j_{t}}(A) .
$$

Proof Similar to the proof of Theorem 3, one may derive the conclusion of this theorem.

\section{The eigenvalue distribution of the Schur complement of nonstrictly diagonally dominant matrices}

In this section, the result of Theorem 2 will be generalized to nonstrictly diagonally dominant matrices.

Theorem 6 Let $A=\left(a_{i j}\right) \in D_{n}$ be nonsingular with real diagonal entries, and $\alpha \subset\langle n\rangle$ such that for all $j \in \alpha^{\prime}=\langle n\rangle-\alpha \subset\langle n\rangle,\left|a_{j j}\right|>R_{j}(A)=\sum_{k=1, k \neq j}^{n}\left|a_{j k}\right|$. Then $A / \alpha$ and $A\left(\alpha^{\prime}\right)$ have the same number of eigenvalues whose real parts are greater (less) than $w$ (resp. $-w)$, where $w$ is defined in (4).

Proof Since $A \in D_{n}$ and is nonsingular, it follows from Lemma 2 that $A(\alpha)$ is nonsingular. Thus, $A / \alpha$ exists. Similar to the proof of Theorem 5 in $[1], \mu[A / \alpha]-w I$ is diagonally dominant coming from (2) in Theorem 1, so is $A / \alpha-w I$. Further, (2) indicates that $\widetilde{a}_{j_{t}, j_{t}}-w>0$ if and only if $\widetilde{a}_{j_{t}, j_{t}}>0$. This implies that $\left|J_{+}(A / \alpha)\right|=\left|J_{+}(A / \alpha-w I)\right|$. Thus, by the Geršgorin theorem, $A / \alpha-w I$ has $\left|J_{+}(A / \alpha)\right|$ eigenvalues with positive real part (on the open right-half complex plane). On the other hand, the eigenvalues of $A / \alpha-w I$ are the eigenvalues of $A / \alpha$ minus $w$, so $A / \alpha$ has $\left|J_{+}(A / \alpha)\right|$ eigenvalues with positive real part greater than $w$. Again, since $[A(\alpha)]^{-1}=[A / \alpha]^{-1}$ (see, e.g., p.184 in [8]) and further that $A / \alpha$ and $[A / \alpha]^{-1}$ have the same number of eigenvalues with positive real part. It follows from Corollary 3 of [1] that $A / \alpha$ and $A\left(\alpha^{\prime}\right)$ have the same number of eigenvalues whose positive real parts are greater than $w$. For the number of negative parts of eigenvalues, the argument above still works with $-A / \alpha$ in place of $A / \alpha$.

Theorem 7 Given a matrix $A=\left(a_{i j}\right) \in D_{n}$ with real diagonal entries, and two sets $\alpha \subset\langle n\rangle$ and $\alpha^{\prime}=\langle n\rangle-\alpha \subset\langle n\rangle$, if

$$
\vartheta=\min _{i \in \alpha, j \in \alpha^{\prime}}\left[\frac{\left|a_{i i}\right|-R_{i}(A)}{\left|a_{i i}\right|} \sum_{k \in \alpha}\left|a_{j k}\right|\right]>0,
$$

then $A / \alpha$ and $A\left(\alpha^{\prime}\right)$ have the same number of eigenvalues whose real parts are greater (less) than $\vartheta($ resp. $-\vartheta)$.

Proof Since $A \in D_{n}$ and $\vartheta=\min _{i \in \alpha, j \in \alpha^{\prime}}\left[\frac{\left|a_{i i}\right|-R_{i}(A)}{\left|a_{i i}\right|} \sum_{k \in \alpha}\left|a_{j k}\right|\right]>0,\left|a_{i i}\right|>R_{i}(A)$ for all $i \in \alpha$ and $A\left(\alpha^{\prime}\right)$ has no diagonally equipotent principal submatrix. Then it follows from Lemma 2 that $A$ is nonsingular, so is $A(\alpha)$. Thus, $A / \alpha$ exists. Similar to the proof of Theorem 6 , the conclusion of this theorem is derived immediately. 


\section{Numerical examples}

In this section, some numerical examples are given to demonstrate the effectiveness of the results obtained in this paper.

Example 1 Consider the following $7 \times 7$ matrix:

$$
A=\left[\begin{array}{ccccccc}
1 & -1 & 0 & 0 & 0 & 0 & 0 \\
1 & -2 & -1 & 0 & 0 & 0 & 0 \\
0 & 1 & -2 & -1 & 0 & 0 & 0 \\
0 & 0 & 1 & -3 & 1 & 0 & 0 \\
0 & 0 & 0 & 1 & 3 & -1 & 0 \\
0 & 0 & 0 & 0 & 1 & -3 & -1 \\
0 & 0 & 0 & 0 & 0 & 1 & 2
\end{array}\right]
$$

Let $\alpha=\{1,2,3\}$. Then $\alpha^{\prime}=\{4,5,6,7\}$. It is verified that $A$ and $A(\alpha)$ are both nonsingular and (13) holds. Thus, $A$ satisfies the conditions of Theorems 3 and 5. According to Theorems 3 and 5, both (2) and (3) hold.

In what follows we will verify the conclusions of Theorems 3 and 5 . Direct computations yield the following results:

$$
\begin{array}{ll}
A / \alpha=\left[\begin{array}{cccc}
-4 & 1 & 0 & 0 \\
1 & 3 & -1 & 0 \\
0 & 1 & -3 & -1 \\
0 & 0 & 1 & 2
\end{array}\right], \\
\omega_{4}=\omega_{5}=\omega_{6}=\omega_{7}=0, & \\
\left|\widetilde{a}_{4,4}\right|-R_{4}(A / \alpha)=3, & \left|a_{4,4}\right|-R_{4}(A)=1, \\
\left|\widetilde{a}_{5,5}\right|-R_{5}(A / \alpha)=1, & \left|a_{5,5}\right|-R_{5}(A)=1, \\
\left|\widetilde{a}_{6,6}\right|-R_{6}(A / \alpha)=1, & \left|a_{6,6}\right|-R_{6}(A)=1, \\
\left|\widetilde{a}_{7,7}\right|-R_{5}(A / \alpha)=1, & \left|a_{7,7}\right|-R_{7}(A)=1, \\
\left|\widetilde{a}_{4,4}\right|+R_{4}(A / \alpha)=5, & \left|a_{4,4}\right|+R_{4}(A)=5, \\
\left|\widetilde{a}_{5,5}\right|+R_{5}(A / \alpha)=5, & \left|a_{5,5}\right|+R_{5}(A)=5, \\
\left|\widetilde{a}_{6,6}\right|+R_{6}(A / \alpha)=5, & \left|a_{6,6}\right|+R_{6}(A)=5, \\
\left|\widetilde{a}_{7,7}\right|+R_{5}(A / \alpha)=5, & \left|a_{7,7}\right|+R_{7}(A)=5 .
\end{array}
$$

Thus,

$$
\begin{aligned}
& \left|\tilde{a}_{4,4}\right|-R_{4}(A / \alpha) \geq\left|a_{4,4}\right|-R_{4}(A)+\omega_{4} \geq\left|a_{4,4}\right|-R_{4}(A) \geq 0, \\
& \left|\tilde{a}_{5,5}\right|-R_{5}(A / \alpha) \geq\left|a_{5,5}\right|-R_{5}(A)+\omega_{5} \geq\left|a_{5,5}\right|-R_{5}(A) \geq 0, \\
& \left|\tilde{a}_{6,6}\right|-R_{6}(A / \alpha) \geq\left|a_{6,6}\right|-R_{6}(A)+\omega_{6} \geq\left|a_{6,6}\right|-R_{6}(A) \geq 0, \\
& \left|\tilde{a}_{7,7}\right|-R_{5}(A / \alpha) \geq\left|a_{7,7}\right|-R_{7}(A)+\omega_{7} \geq\left|a_{7,7}\right|-R_{7}(A) \geq 0
\end{aligned}
$$


and

$$
\begin{aligned}
& \left|\widetilde{a}_{4,4}\right|+R_{4}(A / \alpha) \leq\left|a_{4,4}\right|+R_{4}(A)-\omega_{4} \leq\left|a_{4,4}\right|+R_{4}(A), \\
& \left|\widetilde{a}_{5,5}\right|+R_{5}(A / \alpha) \leq\left|a_{5,5}\right|+R_{5}(A)-\omega_{5} \leq\left|a_{5,5}\right|+R_{5}(A), \\
& \left|\widetilde{a}_{6,6}\right|+R_{6}(A / \alpha) \leq\left|a_{6,6}\right|+R_{6}(A)-\omega_{6} \leq\left|a_{6,6}\right|+R_{6}(A), \\
& \left|\widetilde{a}_{7,7}\right|+R_{5}(A / \alpha) \leq\left|a_{7,7}\right|+R_{7}(A)-\omega_{7} \leq\left|a_{7,7}\right|+R_{7}(A) .
\end{aligned}
$$

(29) and (30) show that the conclusions of Theorems 3 and 5 are true.

Let us investigate Theorem 6 . Since $\alpha=\{1,2,3\}$ and $\alpha^{\prime}=\{4,5,6,7\}$ such that $A$ satisfies the condition of Theorem $6, A / \alpha$ and $A\left(\alpha^{\prime}\right)$ have the same number of eigenvalues whose real parts are greater (less) than $w$ (resp. $-w$ ).

Now, we verify the effectiveness of the conclusion by direct computations. We get $w=1$; further, we have that the eigenvalues of $A / \alpha$ and $A\left(\alpha^{\prime}\right)$ are $-4.127,-2.626,3.000,1.753$ and $-3.106,-2.671,3.023,1.754$, respectively. Thus, $A / \alpha$ and $A\left(\alpha^{\prime}\right)$ have two eigenvalues whose real parts are greater (less) than 1 (resp. -1 ). This shows that the conclusion of Theorem 6 is true.

Example 2 Consider the following $6 \times 6$ matrix:

$$
A=\left[\begin{array}{cccccc}
-5 & -1 & 0 & 1 & 0 & -1 \\
1 & 5 & -1 & 1 & 1 & 0 \\
1 & 1 & 5 & -1 & 0 & 1 \\
-2 & 1 & 1 & -6 & -1 & 1 \\
1 & -2 & -1 & 1 & 6 & -1 \\
-1 & 1 & 1 & 1 & 1 & 5
\end{array}\right] .
$$

Assume $\alpha=\{1,2,3\}$. Then $\alpha^{\prime}=\{4,5,6\}$. By direct computations, we get $\omega_{4}=\omega_{5}=0.80$ and $\omega_{6}=0.60$. Since $\omega_{j_{t}}>0$ for all $j_{t} \in \alpha^{\prime}$, it follows from Theorem 6 that both (2) and (3) hold.

The following will show the effectiveness of Theorem 6 by direct computations. Since

$$
\begin{aligned}
& A / \alpha=\left[\begin{array}{ccc}
-6.50 & -1.25 & 1.25 \\
1.47 & 6.41 & -1.06 \\
0.742 & 0.790 & 5.05
\end{array}\right], \\
& \left|\widetilde{a}_{4,4}\right|-R_{4}(A / \alpha)=4, \quad\left|a_{4,4}\right|-R_{4}(A)=0, \\
& \left|\widetilde{a}_{5,5}\right|-R_{5}(A / \alpha)=3.88, \quad\left|a_{5,5}\right|-R_{5}(A)=0, \\
& \left|\widetilde{a}_{6,6}\right|-R_{6}(A / \alpha)=3.52, \quad\left|a_{6,6}\right|-R_{6}(A)=0, \\
& \left|\widetilde{a}_{4,4}\right|+R_{4}(A / \alpha)=9, \quad\left|a_{4,4}\right|+R_{4}(A)=12, \\
& \left|\widetilde{a}_{5,5}\right|+R_{5}(A / \alpha)=8.94, \quad\left|a_{5,5}\right|+R_{5}(A)=12, \\
& \left|\widetilde{a}_{6,6}\right|+R_{6}(A / \alpha)=6.58, \quad\left|a_{6,6}\right|+R_{6}(A)=10 .
\end{aligned}
$$

Thus,

$$
\left|\widetilde{a}_{4,4}\right|-R_{4}(A / \alpha) \geq\left|a_{4,4}\right|-R_{4}(A)+\omega_{4} \geq\left|a_{4,4}\right|-R_{4}(A) \geq 0,
$$




$$
\begin{aligned}
& \left|\tilde{a}_{5,5}\right|-R_{5}(A / \alpha) \geq\left|a_{5,5}\right|-R_{5}(A)+\omega_{5} \geq\left|a_{5,5}\right|-R_{5}(A) \geq 0, \\
& \left|\widetilde{a}_{6,6}\right|-R_{6}(A / \alpha) \geq\left|a_{6,6}\right|-R_{6}(A)+\omega_{6} \geq\left|a_{6,6}\right|-R_{6}(A) \geq 0
\end{aligned}
$$

and

$$
\begin{aligned}
& \left|\widetilde{a}_{4,4}\right|+R_{4}(A / \alpha) \leq\left|a_{4,4}\right|+R_{4}(A)-\omega_{4} \leq\left|a_{4,4}\right|+R_{4}(A), \\
& \left|\tilde{a}_{5,5}\right|+R_{5}(A / \alpha) \leq\left|a_{5,5}\right|+R_{5}(A)-\omega_{5} \leq\left|a_{5,5}\right|+R_{5}(A), \\
& \left|\widetilde{a}_{6,6}\right|+R_{6}(A / \alpha) \leq\left|a_{6,6}\right|+R_{6}(A)-\omega_{6} \leq\left|a_{6,6}\right|+R_{6}(A) .
\end{aligned}
$$

(33) and (34) show that the conclusions of Theorem 6 are true.

It follows that we will verify the effectiveness of Theorem 7. Direct computations give $\vartheta=0.60>0$. According to Theorem 7, $A / \alpha$ and $A\left(\alpha^{\prime}\right)$ have the same number of eigenvalues whose real parts are greater (less) than $\vartheta$ (resp. $-\vartheta$ ).

By direct computations, eigenvalues of $A / \alpha$ and $A\left(\alpha^{\prime}\right)$ are $-6.422,5.691+0.571 i, 5.691-$ $0.571 i$ and $-5.992,5.496+0.815 i, 5.496-0.815 i$, respectively. As a result, $A / \alpha$ and $A\left(\alpha^{\prime}\right)$ have two eigenvalues whose real parts are greater than 0.6 and have an eigenvalue whose real part is less than -0.6 , respectively. This shows that the conclusion of Theorem 7 is true.

\section{Conclusions}

This mainly studies the disc separation and the eigenvalue location of some special matrices. Firstly, the result on the Geršgorin disc separation from the origin for strictly diagonally dominant matrices and their Schur complements in [1] is extended to nonstrictly diagonally dominant matrices and their Schur complements to reveal that under some conditions the separation of the Schur complement of a nonstrictly diagonally dominant matrix is greater than that of the original grand matrix. Secondly, some significant conclusions are derived to establish the eigenvalue distribution of the Schur complements for nonstrictly diagonally dominant matrices. Finally, some examples are provided to demonstrate the effectiveness of some theoretical results obtained in this paper.

Competing interests

The authors declare that they have no competing interests.

Authors' contributions

All authors contributed equally to this work. All authors read and approved the final manuscript.

\section{Acknowledgements}

The work was supported by the National Natural Science Foundations of China (11501141, 11601409 and 11201362), the Natural Science Foundations of Shaanxi Province of China (2016JM1009), the Foundation of Guizhou Science and Technology Department (Grant No.[2015]2073) and the Natural Science Programs of Education Department of Guizhou Province (Grant No.[2016]066).

\section{Publisher's Note}

Springer Nature remains neutral with regard to jurisdictional claims in published maps and institutional affiliations.

Received: 7 January 2017 Accepted: 22 March 2017 Published online: 05 April 2017

References

1. Liu, JZ, Zhang, FZ: Disc separation of the Schur complement of diagonally dominant matrices and determinantal bounds. SIAM J. Matrix Anal. Appl. 27(3), 665-674 (2005)

2. Liu, JZ, Huang, YQ: Some properties on Schur complements of $H$-matrix and diagonally dominant matrices. Linear Algebra Appl. 389, 365-380 (2004) 
3. Berman, A, Plemmons, RJ: Nonnegative Matrices in the Mathematical Sciences. Academic Press, New York (1979)

4. Bru, R, Corral, C, Gimenez, I, Mas, J: Classes of general H-matrices. Linear Algebra Appl. 429, 2358-2366 (2008)

5. Zhang, $\mathrm{CY}, \mathrm{Luo}, \mathrm{SH}, \mathrm{Xu}, \mathrm{CX}$ : Schur complements of generally diagonally dominant matrices and criterion for irreducibility of matrices. Electron. J. Linear Algebra 18, 69-87 (2009)

6. Zhang, CY, Luo, SH, Xu, FM, Xu, CX: The eigenvalue distribution on Schur complement of nonstrictly diagonally dominant matrices and general H-matrices. Electron. J. Linear Algebra 18, 801-820 (2009)

7. Liu, JZ, Zhang, FZ: The Schur complements of generalized doubly diagonally dominant matrices. Linear Algebra Appl. 378, 231-244 (2004)

8. Zhang, FZ: Matrix Theory: Basic Results and Techniques. Springer, New York (1999)

Submit your manuscript to a SpringerOpen ${ }^{\circ}$ journal and benefit from:

$\rightarrow$ Convenient online submission

Rigorous peer review

- Immediate publication on acceptance

- Open access: articles freely available online

- High visibility within the field

- Retaining the copyright to your article 